\title{
Impulsive control of a class of multiple unstable neural networks
}

\author{
Songfang Jia ${ }^{1 *}$ and Yanheng Chen ${ }^{1}$
}

"Correspondence:

jiasongfang@163.com

${ }^{1}$ College of Mathematic and

Statistics, Chongqing Three Gorges

University, Wanzhou, 404100, China

\section{Springer}

\begin{abstract}
This paper addresses the issue of stability of a class of multiple unstable Cohen-Grossberg neural networks(CGNNs) under impulsive control. Some novel sufficient conditions are given to make the unstable equilibrium points of the model locally $\mu$-stable. An example is offered to demonstrate the effectiveness of the control strategy by comprehensive computer simulations.
\end{abstract}

Keywords: Cohen-Grossberg neural network; Multiple unstable equilibrium points; Impulsive control; $\mu$-stability

\section{Introduction}

Recently, the multi-stability of neural network models has attracted extensive attention because of its wide application in the pattern recognition. Many experts and scholars contributed to this topic (see [1-35]). For example, Cao et al. proved that the CGNNs with multi-stability and multi-periodicity could find $2^{n}$ locally exponentially stable equilibrium points in [1]. The paper [26] revealed the co-existence of unstable and stable equilibrium points of a class of $n$-neuron recurrent neural networks model with time-varying delays. In [31], Nie et al. investigated a class of $n$-neuron competitive neural networks and showed that the systems have exactly $5^{n}$ equilibrium points, and $5^{n}-3^{n}$ among them are unstable. Based on the partition space method, [32] proved that a class of CGNNs with unbounded time-varying delays could have $3^{n}$ equilibrium points, of which $3^{n}-2^{n}$ are unstable and the remaining ones are locally $\mu$-stable. By the above-mentioned references, we can see that most literature focused on the properties of multiple stable equilibrium points of the system. Still, few papers considered the properties of those unstable equilibrium points. Hence it is a challenging problem.

It is common knowledge that impulsive control is a very effective and economical method to address the unstable or chaotic neural networks, and its main idea is to add a pulse into the network topology to control the state of the system. In the past few years, many significant results on impulsive control neural network have been proposed, see [3652]. In [41], the authors studied the delay-dependent passivity analysis of impulsive neural networks by using functional and inequality method and compared the system model with impulsive control and without impulsive control, extended the recent results of passivity. [45] introduced new sandwich control systems with impulse time windows and illustrated

(c) The Author(s) 2021. This article is licensed under a Creative Commons Attribution 4.0 International License, which permits use sharing, adaptation, distribution and reproduction in any medium or format, as long as you give appropriate credit to the original author(s) and the source, provide a link to the Creative Commons licence, and indicate if changes were made. The images or other third party material in this article are included in the article's Creative Commons licence, unless indicated otherwise in a credit line to the material. If material is not included in the article's Creative Commons licence and your intended use is not permitted by statutory regulation or exceeds the permitted use, you will need to obtain permission directly from the copyright holder. To view a copy of this licence, visit http://creativecommons.org/licenses/by/4.0/. 
the stability of the chaotic system by using impulsive. Li et al. in [50] added impulse inputs in unstable neural networks to keep the unstable equilibrium point or the chaotic system stable. Hence it may be a good idea to investigate the stability of unstable equilibrium points of multiple systems by way of impulsive control.

Motivated by the above discussions, we investigated the stability of multiple unstable CGNNs in [32] by introducing a pulse into the system and obtained some sufficient conditions to make unstable equilibrium points of the models locally $\mu$-stable, which generalized the results of paper [50]. The arrangement of this article is as follows. In the second section, the Cohen-Grossberg model and some preliminary conclusions are given. The main results are given and proved in the third section. The corollaries and comparisons with the existing literature are given in the fourth section. Section 5 gives a numerical example with simulation to illustrate the effectiveness of the control strategy. At the end of this paper, the conclusion is made.

\section{System description and preliminaries}

This article focuses on a class of $n$-neuron multiple unstable neural networks under some conditions described by the following equations:

$$
\left\{\begin{array}{l}
\frac{\mathrm{d} x_{i}(t)}{\mathrm{d} t}=-a_{i}\left(x_{i}(t)\right)\left[b_{i}\left(x_{i}(t)\right)-\sum_{j=1}^{n} c_{i j} g_{j}\left(x_{j}(t)\right)-\sum_{j=1}^{n} d_{i j} f_{j}\left(x_{j}(t-\tau(t))\right)+I_{i}\right], \\
\quad i=1,2, \ldots, n, t \geq 0
\end{array}\right.
$$

where $x_{i}(t)$ represents the current state of the $i$ th neuron; $a_{i}\left(x_{i}(t)\right)$ denotes the amplification function of the $i$ th neuron; and $b_{i}\left(x_{i}(t)\right)$ is the inhibition behavior function of the $i$ th neuron; $g_{j}\left(x_{j}(t)\right)$ and $f_{j}\left(x_{j}(t-\tau(t))\right)$ are current activation functions of the $j$ th neuron, and $\tau(t)$ is a nonnegative function and denotes the delay of transmission; $c_{i j}$ is the connection weight of the $i$ th neuron and $j$ th neuron, and $d_{i j}$ denotes their delayed feedback connection weight; $I_{i}$ is a constant and denotes the external input of the $i$ th neuron.

Suppose that model (1) has the initial condition

$$
x_{i}(s)=\varphi_{i}(s), \quad s \in(-\infty, 0],
$$

where $\varphi_{i}(s) \in C((-\infty, 0], \mathbb{R}), i=1,2, \ldots, n$. Let $\left(x_{1}(t), x_{2}(t), \ldots, x_{n}(t)\right)$ and $x^{\star}=\left(x_{1}^{\star}, x_{2}^{\star}, \ldots, x_{n}^{\star}\right)$ stand for a solution and an equilibrium point of model (1), respectively. Then $x^{\star}$ is said to be $\mu$-stable if there exist a positive constant $M$ and a nondecreasing function $\mu(t)$ with $\lim _{t \rightarrow+\infty} \mu(t)=+\infty$ such that

$$
\left|x_{i}(t)-x_{i}^{\star}\right| \leq \frac{M}{\mu(t)}, \quad i=1,2, \ldots, n .
$$

Imitating [32], we can divide the $R^{n}$ into $3^{n}$ non-intersection subregions. Let $\Phi$ be a set of these subregions, and let $(-\infty,+\infty)=\left(-\infty, p_{i}\right) \cup\left[p_{i}, q_{i}\right] \cup\left(q_{i},+\infty\right), i=1,2, \ldots, n$. One can get

$$
\Phi=\left\{\prod_{i=1}^{n} w_{i} \mid w_{i}=\left(-\infty, p_{i}\right),\left[p_{i}, q_{i}\right] \text { or }\left(q_{i},+\infty\right)\right\} .
$$

We define the index subsets for each $\prod_{i=1}^{n} w_{i} \in \Phi$ as $N_{1}=\left\{i \mid w_{i}=\left(-\infty, p_{i}\right), i=1,2, \ldots, n\right\}$, $N_{2}=\left\{i \mid w_{i}=\left[p_{i}, q_{i}\right], i=1,2, \ldots, n\right\}, N_{3}=\left\{i \mid w_{i}=\left(q_{i},+\infty\right), i=1,2, \ldots, n\right\}$, and obviously 
$N_{1} \cup N_{2} \cup N_{3}=\{1,2, \ldots, n\}$. Moreover, we also can separate the set $\Phi$ into two parts $\Phi_{1}$ and $\Phi_{2}$, where $\Phi_{1}=\left\{\prod_{i=1}^{n} w_{i} \mid w_{i}=\left(-\infty, p_{i}\right)\right.$ or $\left.\left(q_{i},+\infty\right), i=1,2, \ldots, n\right\}, \Phi_{2}=\Phi-\Phi_{1}$. Obviously, there are $2^{n}$ and $3^{n}-2^{n}$ elements in $\Phi_{1}$ and $\Phi_{2}$, respectively.

For convenience, let $\hat{A}=\operatorname{diag}\left\{\bar{a}_{1}, \bar{a}_{2}, \ldots, \bar{a}_{n}\right\}$ and $\breve{A}=\operatorname{diag}\left\{\underline{a}_{1}, \underline{a}_{2}, \ldots, \underline{a}_{n}\right\}$ be two positive diagonal matrices. Denote by $a(x(t))=\left(a_{1}\left(x_{1}(t)\right), \ldots, a_{n}\left(x_{n}(t)\right)\right)$ the amplification function of (1), where, for each $i, a_{i}(u)$ is nonnegative continuous and satisfies

$$
\underline{a}_{i} \leq a_{i}(u) \leq \bar{a}_{i}, \quad u \in(-\infty,+\infty), i=1,2, \ldots, n .
$$

Denote by $b(x(t))=\left(b_{1}\left(x_{1}(t)\right), \ldots, b_{n}\left(x_{n}(t)\right)\right)$ the inhibition behavior function, where $b_{i}(u)$ is an odd function that grows monotonically, and there exists a positive matrix $B=$ $\operatorname{diag}\left\{b_{1}, b_{2}, \ldots, b_{n}\right\}$ such that

$$
\frac{b_{i}(y)-b_{i}(z)}{y-z} \geq b_{i}, y, \quad z \in(-\infty,+\infty), y \neq z, i=1,2, \ldots, n .
$$

Denote by $g(x(t))=\left(g_{1}\left(x_{1}(t)\right), \ldots, g_{n}\left(x_{n}(t)\right)\right)$ and $f(x(t))=\left(f_{1}\left(x_{1}(t)\right), \ldots, f_{n}\left(x_{n}(t)\right)\right)$ the activation functions, where $g_{j}(\cdot)$ and $f_{j}(\cdot)$ are continuous linear nondecreasing piecewise function or continuous nonlinear nondecreasing sigmoid function, and one can find some constants $p_{j} \leq q_{j}, m_{j} \leq M_{j}, m_{j}^{\prime} \leq M_{j}^{\prime}, m_{j}^{\prime \prime} \leq M_{j}^{\prime \prime}$, so that

$$
\begin{array}{ll}
m_{j}^{\prime}=\lim _{x \rightarrow-\infty} g_{j}(x), \quad M_{j}^{\prime}=\lim _{x \rightarrow+\infty} g_{j}(x), \quad m_{j}^{\prime \prime}=\lim _{x \rightarrow-\infty} f_{j}(x), \quad M_{j}^{\prime \prime}=\lim _{x \rightarrow+\infty} f_{j}(x) . \\
0 \leq \underline{\sigma}_{j}^{l} \leq \frac{g_{j}(u)-g_{j}(v)}{u-v} \leq \bar{\sigma}_{j}^{l}, \quad 0 \leq \underline{\delta}_{j}^{l} \leq \frac{f_{j}(u)-f_{j}(v)}{u-v} \leq \bar{\delta}_{j}^{l}, \quad \forall u, v \in\left(-\infty, p_{j}\right), \\
0 \leq \underline{\sigma}_{j}^{m} \leq \frac{g_{j}(u)-g_{j}(v)}{u-v} \leq \bar{\sigma}_{j}^{m}, \quad 0 \leq \underline{\delta}_{j}^{m} \leq \frac{f_{j}(u)-f_{j}(v)}{u-v} \leq \bar{\delta}_{j}^{m}, \quad \forall u, v \in\left[p_{j}, q_{j}\right], \\
0 \leq \underline{\sigma}_{j}^{r} \leq \frac{g_{j}(u)-g_{j}(v)}{u-v} \leq \bar{\sigma}_{j}^{r}, \quad 0 \leq \underline{\delta}_{j}^{r} \leq \frac{f_{j}(u)-f_{j}(v)}{u-v} \leq \bar{\delta}_{j}^{r}, \quad \forall u, v \in\left(p_{j},+\infty\right) .
\end{array}
$$

Let $\Sigma^{g}=\operatorname{diag}\left\{\bar{\sigma}_{1}, \bar{\sigma}_{2}, \ldots, \bar{\sigma}_{n}\right\}$ and $\Delta^{f}=\operatorname{diag}\left\{\bar{\delta}_{1}, \bar{\delta}_{2}, \ldots, \bar{\delta}_{n}\right\}$, where $m_{j}=\min \left\{m_{j}^{\prime}, m_{j}^{\prime \prime}\right\}, M_{j}=$ $\min \left\{M_{j}^{\prime}, M_{j}^{\prime \prime}\right\}, \bar{\sigma}_{j}=\max \left\{\bar{\sigma}_{j}^{l}, \bar{\sigma}_{j}^{m}, \bar{\sigma}_{j}^{r}\right\}, \bar{\delta}_{j}=\max \left\{\bar{\delta}_{j}^{l}, \bar{\delta}_{j}^{m}, \bar{\delta}_{j}^{r}\right\}, j=1,2, \ldots, n$. Obviously, both of $\Sigma^{g}$ and $\Delta^{f}$ are two positive matrices.

In addition, we also denote by $C=\left(c_{i j}\right)_{n \times n}$ and $D=\left(d_{i j}\right)_{n \times n}$ the connection weight matrices. Other hypotheses and notations of this article are consistent with the literature [32], no more explanation.

By Theorems 1-3 of paper [32], we know that model (1) has $3^{n}$ equilibrium points, $3^{n}-$ $2^{n}$ among them are unstable, and others are locally $\mu$-stable. Here, we present only the results in [32] as lemmas directly without proof.

Lemma 1 ([32], Theorem 1) For any $\prod_{i=1}^{n} w_{i} \in \Phi$, if

$$
\left\{\begin{array}{l}
-b_{i}\left(p_{i}\right)+c_{i i} g_{i}\left(p_{i}\right)+d_{i j} f_{i}\left(p_{i}\right)+\sum_{\substack{j=1 \\
j \neq i}}^{n} \max \left\{\left(c_{i j}+d_{i j}\right) m_{j},\left(c_{i j}+d_{i j}\right) M_{j}\right\}-I_{i}<0, \\
\quad i \in N_{1} \cup N_{2}, \\
-b_{i}\left(q_{i}\right)+c_{i i} g_{i}\left(q_{i}\right)+d_{i i} f_{i}\left(q_{i}\right)+\sum_{\substack{j=1 \\
j \neq i}}^{n} \min \left\{\left(c_{i j}+d_{i j}\right) m_{j},\left(c_{i j}+d_{i j}\right) M_{j}\right\}-I_{i}>0, \\
\quad i \in N_{2} \cup N_{3},
\end{array}\right.
$$

then there exists at least an equilibrium point of (1) in $\prod_{i=1}^{n} w_{i}$. 
Lemma 2 ([32], Theorem 2) For any $\prod_{i=1}^{n} w_{i} \in \Phi_{1}$, given that

$$
\left\{\begin{array}{l}
-b_{i}\left(p_{i}\right)+c_{i i} g_{i}\left(p_{i}\right)+\sum_{\substack{j=1 \\
j \neq i}}^{n} \max \left\{c_{i j} m_{j}, c_{i j} M_{j}\right\}+\sum_{j=1}^{n} \max \left\{d_{i j} m_{j}, d_{i j} M_{j}\right\}-I_{i}<0, \\
\quad i \in N_{1}, \\
-b_{i}\left(q_{i}\right)+c_{i i} g_{i}\left(q_{i}\right)+\sum_{\substack{j=1 \\
j \neq i}}^{n} \min \left\{c_{i j} m_{j}, c_{i j} M_{j}\right\}+\sum_{j=1}^{n} \min \left\{d_{i j} m_{j}, d_{i j} M_{j}\right\}-I_{i}>0, \\
\quad i \in N_{3},
\end{array}\right.
$$

and the nondecreasing function $\mu(t)>0$ with

$$
\lim _{t \rightarrow+\infty} \mu(t)=+\infty, \quad 0 \leq \sup _{t \geq T^{*}} \frac{\dot{\mu}(t)}{\mu(t)} \leq \alpha, \quad \sup _{t \geq T^{*}} \frac{\mu(t)}{\mu(t-\tau(t))} \leq 1+\beta,
$$

where $\alpha \geq 0, \beta \geq 0$, and $T^{*} \geq 0$. Then $x^{\star}$ is $\mu$-stable in $\prod_{i=1}^{n} w_{i}$ (locally $\mu$-stable in $\left.\Phi_{1}\right)$ if there exist some positive constants $\zeta_{1}, \zeta_{2}, \ldots, \zeta_{n}$ such that

$$
\begin{aligned}
& \left(-\underline{a}_{i} \beta_{i}+\alpha\right) \zeta_{i}+\sum_{j \in N_{1}} \zeta_{j} \bar{a}_{i} \bar{\sigma}_{j}^{l}\left|c_{i j}\right|+\sum_{j \in N_{3}} \zeta_{j} \bar{a}_{i} \bar{\sigma}_{j}^{r}\left|c_{i j}\right| \\
& \quad+(1+\beta)\left(\sum_{j \in N_{1}} \zeta_{j} \bar{a}_{i} \bar{\delta}_{j}^{l}\left|d_{i j}\right|+\sum_{j \in N_{3}} \zeta_{j} \bar{a}_{i} \bar{\delta}_{j}^{r}\left|d_{i j}\right|\right)<0,
\end{aligned}
$$

where $i=1,2, \ldots, n$.

Lemma 3 ([32], Theorem 3) For any $\prod_{i=1}^{n} w_{i} \in \Phi_{2}$, given that (2) holds. If there exist some positive constants $\xi_{1}, \ldots, \xi_{n}$ such that

$$
\begin{gathered}
\min _{i \in N_{2}}\left\{\left(-\beta_{i}+c_{i i} \sigma_{i}^{m}\right) \xi_{i}-\sum_{j \in N_{1}} \xi_{j}\left|c_{i j}\right| \bar{\sigma}_{j}^{l}-\sum_{j \in N_{2}} \xi_{j}\left|c_{i j}\right| \bar{\sigma}_{j}^{m}-\sum_{j \in N_{3}} \xi_{j}\left|c_{i j}\right| \bar{\sigma}_{j}^{r}\right. \\
\left.-\sum_{j \in N_{1}} \xi_{j}\left|d_{i j}\right| \bar{\delta}_{j}^{l}-\sum_{j \in N_{2}} \xi_{j}\left|d_{i j}\right| \bar{\delta}_{j}^{m}-\sum_{j \in N_{3}} \xi_{j}\left|d_{i j}\right| \bar{\delta}_{j}^{r}\right\}>\max \{\lambda, 0\}
\end{gathered}
$$

where

$$
\begin{aligned}
\lambda \triangleq & \max _{i \in N_{1} \cup N_{3}}\left\{\left(-\beta_{i} \xi_{i}+\sum_{j \in N_{1}} \xi_{j}\left|c_{i j}\right| \bar{\sigma}_{j}^{l}+\sum_{j \in N_{2}} \xi_{j}\left|c_{i j}\right| \bar{\sigma}_{j}^{m}+\sum_{j \in N_{3}} \xi_{j}\left|c_{i j}\right| \bar{\sigma}_{j}^{r}\right.\right. \\
& \left.+\sum_{j \in N_{1}} \xi_{j}\left|d_{i j}\right| \bar{\delta}_{j}^{l}+\sum_{j \in N_{2}} \xi_{j}\left|d_{i j}\right| \bar{\delta}_{j}^{m}+\sum_{j \in N_{3}} \xi_{j}\left|d_{i j}\right| \bar{\delta}_{j}^{r}\right\}
\end{aligned}
$$

then $x^{\star}$ in $\prod_{i=1}^{n} w_{i} \in \Phi_{2}$ is unstable.

To discuss the stability under impulsive control of unstable equilibrium points of model (1), the following two lemmas are useful.

Lemma 4 ([11]) Let $Q$ be a positive definite matrix. Then, for any $y, z \in \mathbb{R}^{n}, 2 y^{T} z \leq$ $y^{T} Q^{-1} y+z^{T} Q z$. 
Lemma 5 ([22]) The LMI $Q=\left(\begin{array}{ll}Q_{11} & Q_{12} \\ Q_{12}^{T} & Q_{22}\end{array}\right)<0$ with $Q_{11}=Q_{11}^{T}, Q_{22}=Q_{22}^{T}$ is equivalent to one of the following conditions:

(i) $Q_{22}<0, Q_{11}-Q_{12} Q_{22}^{-1} Q_{12}^{T}<0$.

(ii) $Q_{11}<0, Q_{22}-Q_{12}^{T} Q_{11}^{-1} Q_{12}<0$.

\section{Impulsive control strategy and main results}

For the unstable equilibrium points of model (1), we consider designing an impulsive control strategy to make the unstable equilibrium points stable in each subregion of $\Phi_{2}$. For any subregion $\prod_{i=1}^{n} w_{i} \in \Phi_{2}$, assume that $x^{\star}$ is one unstable equilibrium point in $\prod_{i=1}^{n} w_{i}$ of model (1). Then we introduce the following impulsive control on account of $x^{\star}$ at discrete instances:

$$
\Delta x\left(t_{i}\right)=\Upsilon_{i}\left(x\left(t_{i}^{-}\right)-x^{\star}\right), \quad i \in \mathbb{Z}_{+},
$$

where $\Upsilon_{i} \in \mathbb{R}^{n \times n}$ is a control matrix based on the ith pulse. Let $h(t)=x(t)-x^{\star}$. We can transform (1) and (8) into the matrix equation shown below:

$$
\begin{cases}\frac{\mathrm{d} h(t)}{\mathrm{d} t}=A(h(t))[-B(h(t))+C G(h(t))+D F(h(t-\tau(t)))], & t>0, t \neq t_{i}, \\ \Delta h\left(t_{i}\right)=\Upsilon_{i} h\left(t_{i}^{-}\right), & t=t_{i}, i \in \mathbb{Z}_{+}, \\ h(s)=\phi(s), & s \leq 0,\end{cases}
$$

where $\phi(t)=\varphi(t)-x^{\star}, A(h(t))=a\left(h(t)+x^{\star}\right), B(h(t))=b\left(h(t)+x^{\star}\right)-b\left(x^{\star}\right), G(h(t))=g(h(t)+$ $\left.x^{\star}\right)-g\left(x^{\star}\right), F(h(t))=f\left(h(t)+x^{\star}\right)-f\left(x^{\star}\right)$, and $\lim _{t \rightarrow \infty} \tau(t)=+\infty$.

Definition 1 Let $h(t)$ be a solution to model (9). Then model (9) is said to be locally $\mu$ stable, if one can find a constant $M>0$ satisfying that

$$
\|h\|^{2} \leq \frac{M}{\mu(t)}\|\phi\|^{2}, \quad t \geq 0
$$

where $\|\phi\|^{2}=\sup _{s \leq 0}\|\phi(s)\|^{2}$, and $\mu(t)$ is a continuously differentiable and nondecreasing function on $[0,+\infty)$.

Remark 1 The definition of local $\mu$-stability here includes some famous stabilities such as local asymptotic stability, local Lipschitz stability, and so on. Besides, we design an impulsive control strategy $\left\{t_{i}, \Upsilon_{i}\right\}_{i \in \mathbb{Z}_{+}}$(8) to stabilize the unstable equilibrium points of system (1).

Theorem 1 Suppose that there are two constants $\mu_{1} \geq 1, \mu_{2}>0$ such that

$$
\frac{\mu\left(t_{i}\right)}{\mu\left(t_{i-1}\right)} \leq \mu_{1}, \quad \int_{t_{i-1}}^{t_{i}} \frac{\mu(t)}{\mu^{*}(t-\tau(t))} \mathrm{d} t \leq \mu_{2}, \quad i \in \mathbb{Z}_{+},
$$

where $t_{0}=0$, and $\mu^{*}(t)=\mu(t)$ if $t \geq 0$ and $\mu^{*}(t)=1$ if $t<0$. Besides, one can find a matrix $P>0$, two diagonal matrices $Q_{1}>0, Q_{2}>0$, and three constants $\lambda_{1} \geq 0, \lambda_{2} \geq 0, \gamma>1$ such 
that

$$
\left(\begin{array}{ccc}
\Pi & P \hat{A} C & P \hat{A} D \\
* & -Q_{1} & 0 \\
* & * & -Q_{2}
\end{array}\right) \leq 0, \quad \Delta^{f} Q_{2} \Delta^{f} \leq \lambda_{2} P
$$

and

$$
\ln \mu_{1}+\lambda_{1} \theta+\mu_{2} \lambda_{2} \gamma<\ln \gamma
$$

where $\Pi=-P \check{A} \beta-\beta \check{A} P+\Sigma^{g} Q_{1} \Sigma^{g}-\lambda_{1} P, \theta=\sup _{i \in \mathbb{Z}_{+}}\left\{t_{i}-t_{i-1}\right\}$.

Consider the following positive definite Lyapunov function:

$$
\mathfrak{s}_{P}(t)=\mu^{*} h^{T} P h,
$$

where $h(t)$ is an arbitrary solution in $\prod_{i=1}^{n} w_{i} \in \Phi_{2}$ of model (9) with the initial condition $h(s)=\phi(s), s \leq 0$. If there exist $i \in \mathbb{Z}_{+}$and some $v_{1}<v_{2} \in\left[t_{i-1}, t_{i}\right)$ such that $\{h(t) \mid h(t)=$ $\left.\left(h_{1}(t), h_{2}(t), \ldots, h_{n}(t)\right), t \in\left[v_{1}, v_{2}\right]\right\} \subseteq \prod_{i=1}^{n} w_{i} \in \Phi_{2},\left\{h(t-\tau(t)) \mid h(t)=\left(h_{1}(t), h_{2}(t), \ldots, h_{n}(t)\right)\right.$, $\left.t \in\left[v_{1}, v_{2}\right]\right\} \subseteq \prod_{i=1}^{n} w_{i} \in \Phi_{2}$, and

$$
\mathfrak{s}_{P}(s) \leq \gamma \mathfrak{s}_{P}(t), \quad \forall s \in(-\infty, t]
$$

then

$$
\mathfrak{s}_{P}\left(v_{2}\right) \leq \gamma \mathfrak{s}_{P}\left(v_{1}\right)
$$

Proof For $\forall t \in\left[v_{1}, v_{2}\right]$, by Lemma 3 and Lemma 4, the right upper Dini derivative of function $\mathfrak{s}_{P}(t)$ can be inferred from (11) and (13):

$$
\begin{aligned}
D^{+} \mathfrak{s}_{P}(t)= & \mu^{* \prime}(t) h^{T} P h+2 \mu^{*}(t) h^{T} P h^{\prime} \\
\leq & \mu^{\prime}(t) h^{T} P h+\mu(t)\left[h^{T}(-P \check{A} \beta-\beta \check{A} P) h+h^{T} P \hat{A} C Q_{1} C^{T} \hat{A} P h+h^{T} \Sigma^{g} Q_{1} \Sigma^{g} h\right. \\
& \left.+h^{T} P \hat{A} D Q_{2} D^{T} \hat{A} P h+h^{T}(t-\tau(t)) \Delta^{f} Q_{2} \Delta^{f} h(t-\tau(t))\right] \\
= & \frac{\mu^{\prime}(t)}{\mu(t)} \mu(t) h^{T} P h+\mu(t)\left[h ^ { T } \left(-P \check{A} \beta-\beta \check{A} P+P \hat{A} C Q_{1} C^{T} \hat{A} P+\Sigma^{g} Q_{1} \Sigma^{g}\right.\right. \\
& \left.\left.+P \hat{A} D Q_{2} D^{T} \hat{A} P\right) h+h^{T}(t-\tau(t)) \Delta^{f} Q_{2} \Delta^{f} h(t-\tau(t))\right] \\
\leq & \frac{\mu^{\prime}(t)}{\mu(t)} \mu(t) h^{T} P h+\mu(t)\left[h^{T} \lambda_{1} P h+h^{T}(t-\tau(t)) \lambda_{2} P h(t-\tau(t))\right] \\
= & \left(\frac{\mu^{\prime}(t)}{\mu(t)}+\lambda_{1}\right) \mu(t) h^{T} P h+\lambda_{2} \frac{\mu(t)}{\mu^{*}(t-\tau(t))} \mu^{*}(t-\tau(t)) h^{T}(t-\tau(t)) P h(t-\tau(t)) \\
= & \left(\frac{\mu^{\prime}(t)}{\mu(t)}+\lambda_{1}\right) \mathfrak{s}_{P}(t)+\lambda_{2} \frac{\mu(t)}{\mu^{*}(t-\tau(t))} \mathfrak{s}_{P}(t-\tau(t)) \\
\leq & \left(\frac{\mu^{\prime}(t)}{\mu(t)}+\lambda_{1}+\lambda_{2} \gamma \frac{\mu(t)}{\mu^{*}(t-\tau(t))}\right) \mathfrak{s}_{P}(t) .
\end{aligned}
$$


Let us take the integral of (14) for $t$ in the interval $\left[v_{1}, v_{2}\right]$. By (10) and (12), it can be obtained that

$$
\begin{aligned}
\ln \frac{\mathfrak{s}_{P}\left(v_{2}\right)}{\mathfrak{s}_{P}\left(v_{1}\right)} & \leq \ln \frac{\mu\left(t_{i}\right)}{\mu\left(t_{i-1}\right)}+\lambda_{1} \theta+\lambda_{2} \gamma \int_{t_{k-1}}^{t_{k}} \frac{\mu(t)}{\mu^{*}(t-\tau(t))} \mathrm{d} t \\
& \leq \ln \mu_{1}+\lambda_{1} \theta+\lambda_{2} \gamma \mu_{2} \leq \ln \gamma .
\end{aligned}
$$

Therefore, one can get $\mathfrak{s}_{P}\left(v_{2}\right) \leq \gamma \mathfrak{s}_{P}\left(v_{1}\right)$ from (15).

Theorem 2 Let $x^{\star}$ be one unstable equilibrium point in $\prod_{i=1}^{n} w_{i} \in \Phi_{2}$ of model (1). If (10), (11), and (12) hold and

$$
\gamma\left(I+\Upsilon_{i}\right)^{T} P\left(I+\Upsilon_{i}\right) \leq P, \quad i \in \mathbb{Z}_{+},
$$

then model (9) is locally $\mu$-stable. Furthermore, $x^{\star}$ under impulsive control strategy $\left\{t_{i}, \Upsilon_{i}\right\}_{i \in \mathbb{Z}_{+}}$(8) is locally $\mu$-stable, and so model (1) can increase $3^{n}-2^{n}$ locally $\mu$-stable equilibrium points.

Proof We use a similar method as that in [50] to prove the theorem. Let $\phi \neq 0$. Then we just have to prove the following inequality:

$$
\mathfrak{s}_{P}(t) \leq \gamma M, \quad t \in\left[t_{i-1}, t_{i}\right), i \in \mathbb{Z}_{+},
$$

where $M=\mu(0) \lambda_{\max }(P)\|\phi\|^{2},\left\{h(t) \mid h(t) \in R^{n}, t \in\left[t_{i-1}, t_{i}\right)\right\} \subset \prod_{i=1}^{n} w_{i}$ in $\Phi_{2}$. Note that

$$
\mathfrak{s}_{P}(t)=\mu^{*} h^{T} P h=h^{T} P h \leq \mu(0) \lambda_{\max }(P)\|\phi\|^{2} \leq \gamma M, \quad t \leq 0 .
$$

Firstly, if $\mathfrak{s}_{P}(t) \leq \gamma M$ is not true when $k=1$, then there is $v_{2} \in\left(0, t_{1}\right)$ so that $h\left(v_{2}\right) \in$ $\prod_{i=1}^{n} w_{i}$, and then

$$
\mathfrak{s}_{P}\left(v_{2}\right)=\gamma M, \quad \mathfrak{s}_{P}(t) \leq \gamma M, \quad t \leq v_{2} .
$$

By (18) and (19), it can be seen that there must exist $v_{1} \in\left[0, v_{2}\right)$ and $h\left(v_{1}\right) \in \prod_{i=1}^{n} w_{i}$ in $\Phi_{2}$ so that

$$
\mathfrak{s}_{P}\left(v_{1}\right)=M, \quad M \leq \mathfrak{s}_{P}(t) \leq \gamma M, \quad t \in\left[v_{1}, v_{2}\right] .
$$

For any $t \in\left[v_{1}, v_{2}\right]$, by (19) and (20), it follows that

$$
\mathfrak{s}_{P}(s) \leq \gamma M \leq \gamma \mathfrak{s}_{P}(t), \quad \forall s \in(-\infty, t] .
$$

Meanwhile, by Theorem 1, we can get

$$
\gamma M=\mathfrak{s}_{P}\left(v_{2}\right)<\gamma \mathfrak{s}_{P}\left(v_{1}\right)=\gamma M,
$$

which is a contradiction. Hence $\mathfrak{s}_{P}(t) \leq \gamma M$, and $k=1$ holds. 
Secondly, suppose that $\mathfrak{s}_{P}(t) \leq \gamma M$ holds for any $k \leq N, \forall N \in \mathbb{Z}_{+}$. However, if $\mathfrak{s}_{P}(t) \leq$ $\gamma M$ is not true when $n=N+1$, then there exists $v_{2}^{*} \in\left(t_{N}, t_{N+1}\right)$ such that $h\left(v_{2}^{*}\right) \in \prod_{i=1}^{n} w_{i}$, and then

$$
\mathfrak{s}_{P}\left(v_{2}^{*}\right)=\gamma M, \quad \mathfrak{s}_{P}(t) \leq \gamma M, \quad t \leq v_{2}^{*} .
$$

With respect to (16), we can obtain that

$$
\begin{aligned}
\mathfrak{s}_{P}\left(t_{N}\right) & =\mu^{*}\left(t_{N}\right) h^{T}\left(t_{N}\right) P h\left(t_{N}\right)=\mu^{*} h^{T}\left(t_{N}^{-}\right)\left(I+\Upsilon_{N}\right)^{T} P\left(I+\Upsilon_{N}\right) h\left(t_{N}^{-}\right) \\
& \leq \frac{1}{\gamma} \mathfrak{s}_{P}\left(t_{N}^{-}\right)=M .
\end{aligned}
$$

By (22) and (23), there must exist $v_{1}^{*} \in\left[t_{N}, v_{2}^{*}\right)$ so that $h\left(v_{1}^{*}\right) \in \prod_{i=1}^{n} w_{i}$, and then

$$
\mathfrak{s}_{P}\left(v_{1}^{*}\right)=M, \quad M \leq \mathfrak{s}_{P}(t) \leq \gamma M, \quad t \in\left[v_{1}^{*}, v_{2}^{*}\right] .
$$

Furthermore, for any $t \in\left[v_{1}^{*}, v_{2}^{*}\right]$, by (23) and (24), we have

$$
\mathfrak{s}_{P}(s) \leq \gamma M \leq \gamma \mathfrak{s}_{P}(t), \quad \forall s \in(-\infty, t]
$$

However, we can obtain by Theorem 1

$$
\gamma M=\mathfrak{s}_{P}\left(v_{2}^{*}\right)<\gamma \mathfrak{s}_{P}\left(v_{1}^{*}\right)=\gamma M
$$

which leads to a contradiction. Thus $\mathfrak{s}_{p}(t) \leq \gamma M$, and $k=N+1$ holds.

Finally, by mathematical induction, we get

$$
\begin{aligned}
& \mu(t) \lambda_{\min }(P) h^{T} h \leq \mu^{*}(t) h^{T} P h=\mathfrak{s}_{P}(t) \leq \gamma \mu(0) \lambda_{\max }(P)\|\varphi\|^{2}, \\
& \forall t \in\left[t_{i-1}, t_{i}\right), i \in \mathbb{Z}_{+},
\end{aligned}
$$

which implies that (26) satisfies Definition 1. Hence model (9) is $\mu$-stable in $\prod_{i=1}^{n} w_{i}$ of $\Phi_{2}$. Consequently, $x^{\star}$ under impulsive control (8) is locally $\mu$-stable, and so model (1) can add $3^{n}-2^{n}$ locally $\mu$-stable points.

Remark 2 Theorem 2 shows that the impulse control can make the unstable regions stable and also increases the stable equilibrium points of model (1).

\section{Corollaries and comparisons}

On the basis of lemmas and theorems above, the following conclusions are drawn and compared with those in the existing literature.

When $a_{i}\left(x_{i}(t)\right)=1$, model (1) converts into the model HNN:

$$
\left\{\begin{array}{l}
\frac{\mathrm{d} x_{i}(t)}{\mathrm{d} t}=-b_{i}\left(x_{i}(t)\right)+\sum_{j=1}^{n} c_{i j} g_{j}\left(x_{j}(t)\right)+\sum_{j=1}^{n} d_{i j} f_{j}\left(x_{j}(t-\tau(t))\right)-I_{i}, \\
\quad i=1,2, \ldots, n, t \geq 0 .
\end{array}\right.
$$

According to conditions (2)-(6), there are at least $3^{n}$ equilibrium points in model (27), $2^{n}$ of them in $\Phi_{1}$ are locally $\mu$-stable, and the remaining in $\Phi_{2}$ are unstable. 
Let $h(t)=x(t)-x^{\star}, t \geq 0$, where $x^{\star}$ is an unstable equilibrium point in $\prod_{i=1}^{n} w_{i} \in \Phi_{2}$ and $x(t)$ is a solution of (27) with the initial condition $x(s)=\varphi(s) \in \Phi_{2}, s \in(-\infty, 0]$. Then model (27) and the impulsive control (8) with respect to $x^{\star}$ can be turned into

$$
\begin{cases}\frac{\mathrm{d} h(t)}{\mathrm{d} t}=-B(h(t))+C G(h(t))+D F(h(t-\tau(t))), & t>0, t \neq t_{i}, \\ h(t)=\phi(t), & t \leq 0, \\ \Delta h\left(t_{i}\right)=\Upsilon_{i} h\left(t_{i}^{-}\right), & t=t_{i}, i \in \mathbb{Z}_{+} .\end{cases}
$$

Corollary 1 Under conditions (10), (12), (16), and

$$
\left(\begin{array}{ccc}
\Pi & P C & P D \\
* & -Q_{1} & 0 \\
* & * & -Q_{2}
\end{array}\right) \leq 0, \quad \Delta^{f} Q_{2} \Delta^{f} \leq \lambda_{2} P
$$

where $\Pi=-P \beta-\beta P+\Sigma^{g} Q_{1} \Sigma^{g}-\lambda_{1} P$, model (28) is $\mu$-stable in $\prod_{i=1}^{n} w_{i}$. Furthermore, $x^{\star}$ under impulsive control strategy $\left\{t_{i}, \Upsilon_{i}\right\}_{i \in \mathbb{Z}_{+}}$(8) is locally $\mu$-stable, and so model (27) can increase $3^{n}-2^{n}$ locally $\mu$-stable points.

When $a_{i}\left(x_{i}(t)\right)=1$ and $b_{i}\left(x_{i}(t)\right)=b_{i} \cdot x_{i}(t)$, model (1) changes into

$$
\left\{\begin{array}{l}
\frac{\mathrm{d} x_{i}(t)}{\mathrm{d} t}=-b_{i} \cdot x_{i}(t)+\sum_{j=1}^{n} c_{i j} g_{j}\left(x_{j}(t)\right)+\sum_{j=1}^{n} d_{i j} f_{j}\left(x_{j}(t-\tau(t))\right)-I_{i}, \\
i=1,2, \ldots, n, t \geq 0 .
\end{array}\right.
$$

If (30) meets (4) and the following conditions (31)-(33):

$$
\begin{aligned}
& \left\{\begin{array}{l}
-b_{i} p_{i}+c_{i i} g_{i}\left(p_{i}\right)+\sum_{\substack{j=1 \\
j \neq i}}^{n} \max \left\{c_{i j} m_{j}, c_{i j} M_{j}\right\}+\sum_{j=1}^{n} \max \left\{d_{i j} m_{j}, d_{i j} M_{j}\right\}-I_{i}<0, \\
-b_{i} q_{i}+c_{i i} g_{i}\left(q_{i}\right)+\sum_{\substack{j=1 \\
j \neq i}}^{n} \min \left\{c_{i j} m_{j}, c_{i j} M_{j}\right\}+\sum_{j=1}^{n} \min \left\{d_{i j} m_{j}, d_{i j} M_{j}\right\}-I_{i}>0, \\
i=1,2, \ldots, n .
\end{array}\right. \\
& \left(-\underline{a}_{i} \beta_{i}+\alpha\right) \zeta_{i}+\sum_{j \in N_{1}} \zeta_{j} \bar{a}_{i} \bar{\sigma}_{j}^{l}\left|c_{i j}\right|+\sum_{j \in N_{3}} \zeta_{j} \bar{a}_{i} \bar{\sigma}_{j}^{r}\left|c_{i j}\right| \\
& +(1+\beta)\left(\sum_{j \in N_{1}} \zeta_{j} \bar{a}_{i} \bar{\delta}_{j}^{l}\left|d_{i j}\right|+\sum_{j \in N_{3}} \zeta_{j} \bar{a}_{i} \bar{\delta}_{j}^{r}\left|d_{i j}\right|\right)<0, \\
& \min _{i \in N_{2}}\left\{\left(-b_{i}+c_{i i} \sigma_{i}^{m^{*}}\right) \xi_{i}-\sum_{j \in N_{1}} \xi_{j}\left|c_{i j}\right| \bar{\sigma}_{j}^{l}-\sum_{j \in N_{2}} \xi_{j}\left|c_{i j}\right| \bar{\sigma}_{j}^{m}-\sum_{j \in N_{3}} \xi_{j}\left|c_{i j}\right| \bar{\sigma}_{j}^{r}-\sum_{j \in N_{1}} \xi_{j}\left|d_{i j}\right| \bar{\delta}_{j}^{l}\right. \\
& \left.-\sum_{j \in N_{2}} \xi_{j}\left|d_{i j}\right| \bar{\delta}_{j}^{m}-\sum_{j \in N_{3}} \xi_{j}\left|d_{i j}\right| \bar{\delta}_{j}^{r}\right\}>\max \{\lambda, 0\},
\end{aligned}
$$

where $\zeta_{1}, \zeta_{2}, \ldots, \zeta_{n}, \xi_{1}, \xi_{2}, \ldots, \xi_{n}$ are positive constants, and

$$
\begin{aligned}
\lambda \triangleq & \max _{i \in N_{1} \cup N_{3}}\left\{\left(-b_{i} \xi_{i}+\sum_{j \in N_{1}} \xi_{j}\left|c_{i j}\right| \bar{\sigma}_{j}^{l}+\sum_{j \in N_{2}} \xi_{j}\left|c_{i j}\right| \bar{\sigma}_{j}^{m}+\sum_{j \in N_{3}} \xi_{j}\left|c_{i j}\right| \bar{\sigma}_{j}^{r}\right.\right. \\
& \left.+\sum_{j \in N_{1}} \xi_{j}\left|d_{i j}\right| \bar{\delta}_{j}^{l}+\sum_{j \in N_{2}} \xi_{j}\left|d_{i j}\right| \bar{\delta}_{j}^{m}+\sum_{j \in N_{3}} \xi_{j}\left|d_{i j}\right| \bar{\delta}_{j}^{r}\right\}
\end{aligned}
$$


then one can obtain that there exist at least $3^{n}$ equilibrium points in model (30), $3^{n}-2^{n}$ of them in $\Phi_{2}$ are unstable, and the remaining $2^{n}$ points in $\Phi_{1}$ are locally $\mu$-stable.

Let $x^{\star}$ be an unstable equilibrium point in $\prod_{i=1}^{n} w_{i} \in \Phi_{2}$ and $x(t)$ be a solution of (30) with the initial condition $x(s)=\varphi(s) \in \Phi_{2}, s \in(-\infty, 0]$, and let $h(t)=x(t)-x^{\star}, t \geq 0$. Then model (30) and the impulsive control (8) with respect to $x^{\star}$ can transform into the following matrix form:

$$
\begin{cases}\frac{\mathrm{d} h(t)}{\mathrm{d} t}=-B h(t)+C G(h(t))+D F(h(t-\tau(t))), & t>0, t \neq t_{i}, \\ h(t)=\phi(t), & t \leq 0, \\ \Delta h\left(t_{i}\right)=\Upsilon_{i} h\left(t_{i}^{-}\right), & t=t_{i}, i \in \mathbb{Z}_{+} .\end{cases}
$$

Corollary 2 Under conditions (10),(12),(16), and (29), where $\Pi=-P B-B P+\Sigma^{g} Q_{1} \Sigma^{g}-$ $\lambda_{1} P$, model (35) is $\mu$-stable in $\prod_{i=1}^{n} w_{i}$. Furthermore, $x^{\star}$ under impulsive control strategy $\left\{t_{i}, \Upsilon_{i}\right\}_{i \in \mathbb{Z}_{+}}(8)$ is locally $\mu$-stable, and so model (30) can increase $3^{n}-2^{n}$ locally $\mu$-stable points.

Remark 3 The net self-inhibition function $b_{i}\left(x_{i}(t)\right)$ in model (30) is monotone increasing and odd, which contains the case of Ref. [50]. Hence model (30) is more general.

Remark 4 Ref. [50] studied the stability of unstable systems with unbounded time-varying delays at some certain discrete time for HNN model (30) and derived some control results to stabilize neural networks with an unstable equilibrium point by the impulsive control. However, we studied in the present paper the stability of multiple unstable equilibrium points.

Corollary 3 When $\mu(t)=1+\varsigma t, \varsigma>0$, and $\tau(t)=k t, k \in(0,1)$, assume that (2), (3), (6) hold in $\Phi_{1}$, and satisfy

$$
\begin{aligned}
& \left(-\underline{a}_{i} \beta_{i}+\varsigma\right) \zeta_{i}+\sum_{j \in N_{1}} \zeta_{j} \bar{a}_{i} \bar{\sigma}_{j}^{l}\left|c_{i j}\right|+\sum_{j \in N_{3}} \zeta_{j} \bar{a}_{i} \bar{\sigma}_{j}^{r}\left|c_{i j}\right| \\
& +(1+\varsigma \tau)\left(\sum_{j \in N_{1}} \zeta_{j} \bar{a}_{i} \bar{\delta}_{j}^{l}\left|d_{i j}\right|+\sum_{j \in N_{3}} \zeta_{j} \bar{a}_{i} \bar{\delta}_{j}^{r}\left|d_{i j}\right|\right)<0 .
\end{aligned}
$$

Let $\ln (1+\varsigma \theta)+\lambda_{1} \theta+\frac{\theta}{1-\tau} \lambda_{2} \gamma<\ln \gamma$.If (11) and (16) hold for $\Phi_{2}$, then model (1) under the impulsive control (8) is asymptotically stable in each local region of $\Phi_{2}$.

Corollary 4 When $\mu(t)=\ln (f+t), f>e$, and $\tau(t)=t-\ln t / t$, assume that (2), (3), (6) hold in $\Phi_{1}$ and satisfy

$$
-\underline{a}_{i} \beta_{i} \zeta_{i}+\sum_{j \in N_{1}} \zeta_{j} \bar{a}_{i} \bar{\sigma}_{j}^{l}\left|c_{i j}\right|+\sum_{j \in N_{3}} \zeta_{j} \bar{a}_{i} \bar{\sigma}_{j}^{r}\left|c_{i j}\right|+\left(\sum_{j \in N_{1}} \zeta_{j} \bar{a}_{i} \bar{\delta}_{j}^{l}\left|d_{i j}\right|+\sum_{j \in N_{3}} \zeta_{j} \bar{a}_{i} \bar{\delta}_{j}^{r}\left|d_{i j}\right|\right)<0 .
$$

Let $\ln \frac{\ln (f+\theta)}{\ln f}+\lambda_{1} \theta+\ln (1+\theta / f) \lambda_{2} \gamma<\ln \gamma$.If (11) and (16) hold for $\Phi_{2}$, then model (1) under impulsive control (8) is log-stable in each local region of $\Phi_{2}$. 
Corollary 5 When $\mu(t)=\ln (f+t), \tau(t)=(f+t)-(f+t)^{\epsilon}$, where $f>e, \epsilon \in(0,1)$, assume that (2), (3), (6) hold in $\Phi_{1}$ and satisfy

$$
-\underline{a}_{i} \beta_{i} \zeta_{i}+\sum_{j \in N_{1}} \zeta_{j} \bar{a}_{i} \bar{\sigma}_{j}^{l}\left|c_{i j}\right|+\sum_{j \in N_{3}} \zeta_{j} \bar{a}_{i} \bar{\sigma}_{j}^{r}\left|c_{i j}\right|+\frac{1}{\epsilon}\left(\sum_{j \in N_{1}} \zeta_{j} \bar{a}_{i} \bar{\delta}_{j}^{l}\left|d_{i j}\right|+\sum_{j \in N_{3}} \zeta_{j} \bar{a}_{\bar{i}} \bar{\delta}_{j}^{r}\left|d_{i j}\right|\right)<0 .
$$

Let $\ln \frac{\ln (f+\theta)}{\ln f}+\lambda_{1} \theta+\frac{\theta}{\epsilon} \lambda_{2} \gamma<\ln \gamma$.If(11) and (16) hold for $\Phi_{2}$, then model (1) under impulsive control (8) is log-log-stable in each region of $\Phi_{2}$.

\section{Numerical example}

Example Consider the following two-dimensional CGNNs model:

$$
\left\{\begin{array}{l}
\frac{\mathrm{d} x_{i}(t)}{\mathrm{d} t}=-a\left(x_{i}(t)\right)\left[b_{i}\left(x_{i}(t)\right)-\sum_{j=1}^{n} c_{i j} g\left(x_{j}(t)\right)-\sum_{j=1}^{n} d_{i j} f\left(x_{j}(t-\tau(t))\right)+I_{i}\right], \\
\quad i=1,2, t \geq 0,
\end{array}\right.
$$

where $a(x)=1+0.2 \sin (x), b_{1}\left(x_{1}(t)\right)=x_{1}(t), b_{2}\left(x_{2}(t)\right)=-1.2 x_{2}(t), f(x)=\frac{|x+1|-|x-1|}{2}$,

$$
\begin{aligned}
& g(x)= \begin{cases}\tanh (0.2 x)-\tanh (1)+\tanh (0.2), & x<-1, \\
\tanh (x), & -1 \leq x \leq 1, \quad \tau(t)=0.2 t, \\
\tanh (0.2 x)+\tanh (1)-\tanh (0.2), & x>1,\end{cases} \\
& C=\left(c_{i j}\right)=\left(\begin{array}{cc}
3.5 & 0.2 \\
0.4 & 4.8
\end{array}\right), \quad D=\left(d_{i j}\right)=\left(\begin{array}{cc}
0.4 & 0.2 \\
0.2 & 0.5
\end{array}\right), \quad I_{1}=I_{2}=\left(\begin{array}{l}
-0.3 \\
-0.6
\end{array}\right) \text {. }
\end{aligned}
$$

Let $\mu^{*}(t)=1+0.2 t$ if $t \geq 0$ and $\mu^{*}(t)=1$ if $t<0$. Then we know that the hypothesis of Eq. (36) and $\mu^{*}(t)$ satisfy condition (6) by calculation. Therefore, by Lemmas $1-3$, there are nine equilibrium points in model (36), four of which are $\mu$-stable, and others are unstable. Running program $[x, f v a l]=f$ solve $\left(' m y f u n 7^{\prime}, x 0\right)$ with Matlab software for model (36) in each subregion, one can obtain the nine equilibrium points of (36) as follows:

$$
\begin{array}{ll}
x(1)=(-3.8676,5.9164)^{T}, & x(2)=(-0.2792,6.4788)^{T}, \\
x(3)=(6.1086,7.4167)^{T}, & x(4)=(-4.6061,0.0284)^{T}, \\
x(5)=(-0.0852,-0.1349)^{T}, & x(6)=(5.2870,-0.3416)^{T}, \\
x(7)=(-5.3083,-6.1639)^{T}, & x(8)=(0.0586,-5.2811)^{T}, \\
x(9)=(4.8374,-4.3921)^{T} . &
\end{array}
$$

Trace the solutions of model (36) with 150 initial conditions, the dynamics of $x_{1}(t)$ and $x_{2}(t)$ are depicted in the above three graphs of Fig. 1, which show that there are four locally $\mu$-stable equilibrium points, which is in accord with our results.

With the functions and parameters given above, we can find that $\check{A}=\operatorname{diag}\{0.8,0.8\}$, $\hat{A}=\operatorname{diag}\{1.2,1.2\}, \Sigma^{g}=\operatorname{diag}\{1.564,1.564\}, \Delta^{f}=\operatorname{diag}\{1,1\}, \lambda_{1}=18$. And we can obtain the following results by resorting to Matlab LMI control toolbox:

$$
P=\left(\begin{array}{ll}
0.4605 & 0.0069 \\
0.0069 & 0.4558
\end{array}\right), \quad Q_{1}=\left(\begin{array}{cc}
1.7450 & 0 \\
0 & 1.7450
\end{array}\right)
$$



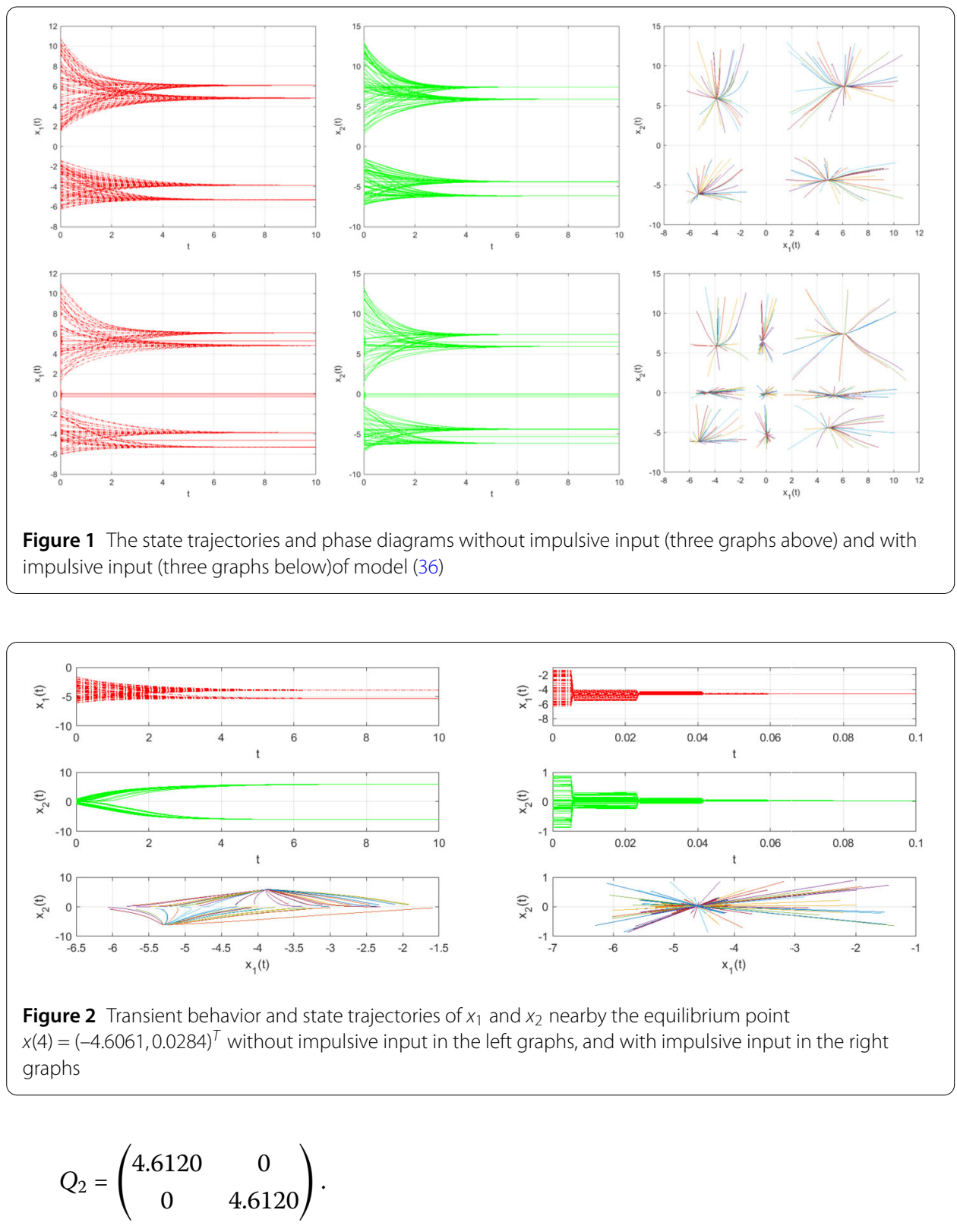

Let $\lambda_{2}=11, \gamma=3.5, \theta=0.0186$, and $t_{i}=0.018 i$. Then we can deduce the following impulsive control matrix by (16):

$$
\Upsilon_{i}=\left(\begin{array}{cc}
-0.7 & 0 \\
0 & -0.7
\end{array}\right)
$$

Under the impulsive control matrix (37), the state trajectory curve of model (36) can be obtained with the same 150 initial solutions, which is the below three graphs of Fig. 1. It is easy to get that the stable equilibrium points of model (36) are more than before adding impulse, and just right one equilibrium point exists in each region of model (36). Specifically, Figs. 2-6 show that the other five equilibrium points are unstable, while they are locally $\mu$-stable after adding impulse, which verifies the effectiveness of the control strategy and the correctness of the obtained results. 

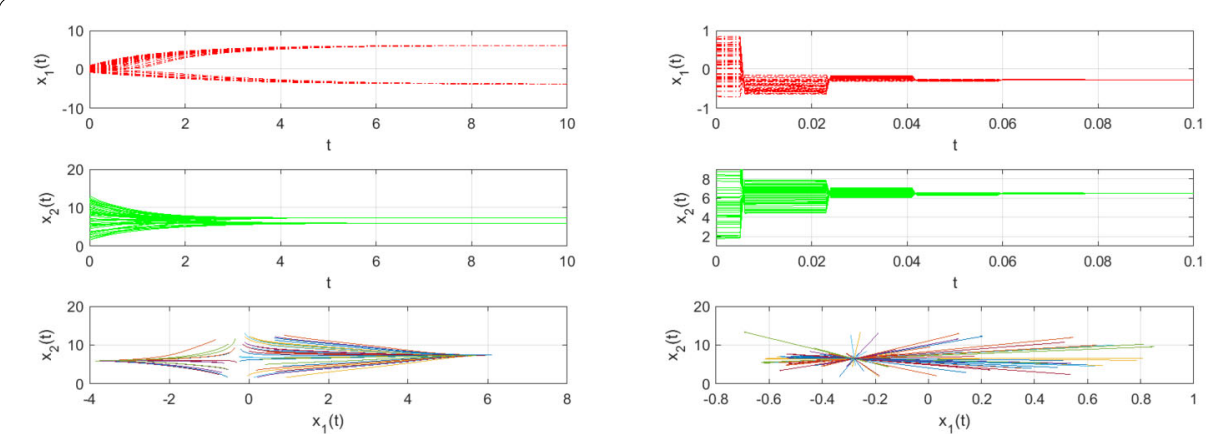

Figure 3 Transient behavior and state trajectories of $x_{1}$ and $x_{2}$ near the equilibrium point $x(2)=(-0.2792,6.4788)^{T}$ without impulsive input in the left graphs, and with impulsive input in the right graphs
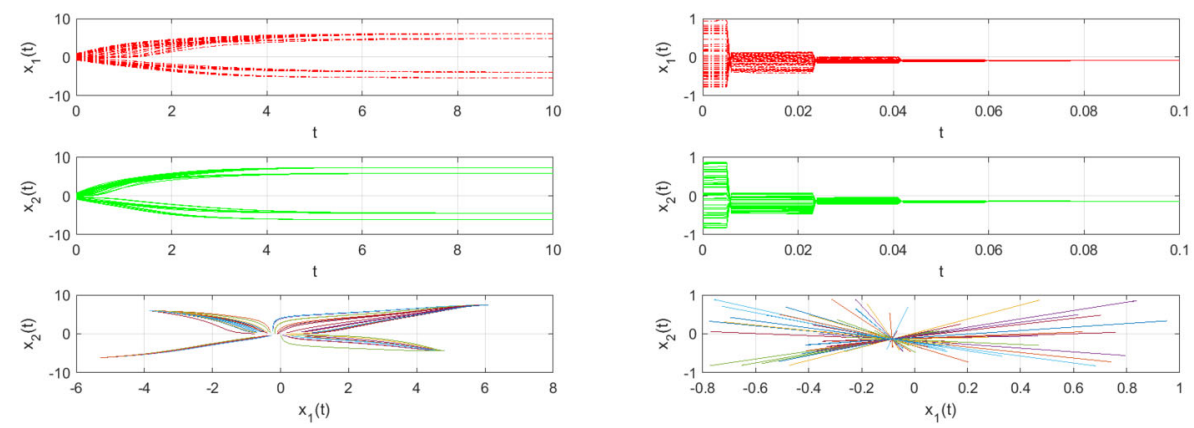

Figure 4 Transient behavior and state trajectories of $x_{1}$ and $x_{2}$ near the equilibrium point $x(5)=(-0.0852,-0.1349)^{T}$ without impulsive input in the left graphs, and with impulsive input in the right graphs

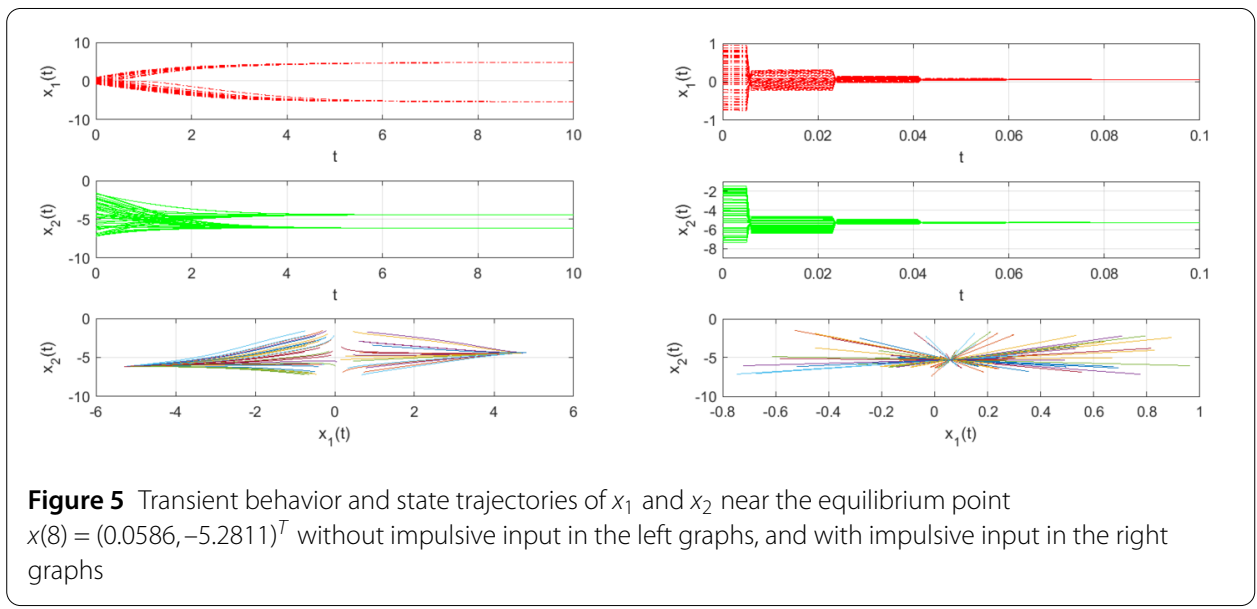

Remark 5 The activation functions in the example of Ref. [50] without time delay and with time delay are the same, but they are different in the present paper. Therefore, the simulation of this paper is closer to the results of the theory. 

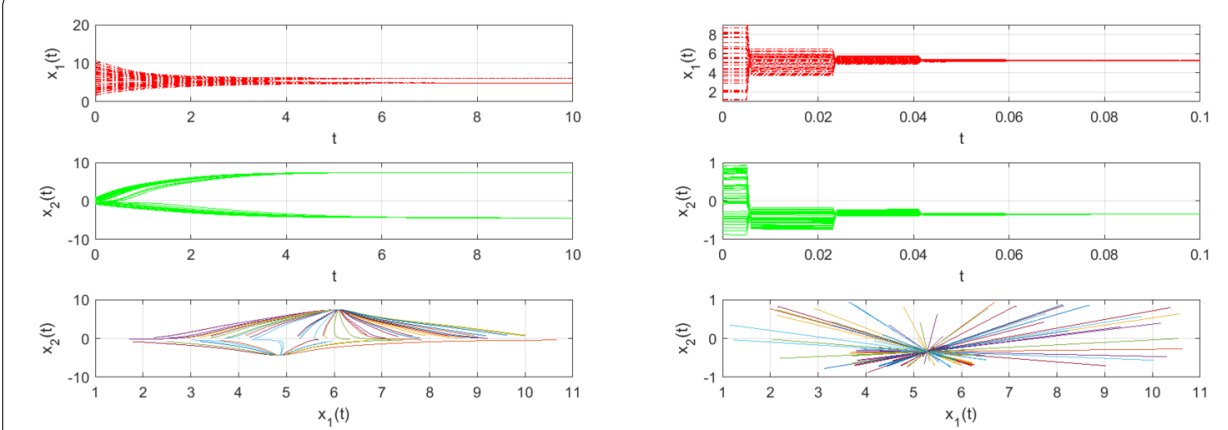

Figure 6 Transient behavior and state trajectories of $x_{1}$ and $x_{2}$ near the equilibrium point $x(6)=(5.2870,-0.3416)^{T}$ without impulsive input in the left graphs, and with impulsive input in the right graphs

\section{Conclusion}

Impulsive control of multiple unstable CGNNs with unbounded time-varying delays is studied in this article. Ref. [32] proved that there exist multiple equilibrium points, and some of them are unstable in model(1). For those unstable equilibrium points, we introduce an impulsive control strategy into the unstable region to ensure that system (1) is $\mu$-stable in each local region of $\Phi_{2}$. In Sect. 4, we conclude some results of other models and point out the advantages of model (28). Meanwhile, we summarize that model (1) is $\mu$-stable in each local region of $R^{n}$ under impulsive control, including the asymptotically stable, log-stable, and log-log-stable. In addition, we also show the effectiveness of impulsive control strategy by one example and its comprehensive numerical simulations. From the results of this article, we see that it is an effective method to study the stability of multiple unstable CGNNs by introducing impulse inputs. Therefore, we can investigate the stability of other multiple unstable system by employing the impulsive control strategy further.

\section{Acknowledgements}

The authors would like to thank the referees for their valuable comments and suggestion.

\section{Funding}

This work was supported in part by the Scientific and Technological Research Program of Chongqing Municipal Education Commission (Grant No. KJQN202001217), the Foundation of Chongqing Three Gorges University (Grant No. 18ZDPY07), Chongqing Municipal Key Laboratory of Institutions of Higher Education (Grant No.[2017]3), Program of Chongqing Development and Reform Commission (Grant No. 2017[1007]).

\section{Availability of data and materials}

Not applicable.

\section{Competing interests}

The authors declare that they have no competing interests.

\section{Authors' contributions}

The authors contributed equally to the writing of this paper. The authors read and approved the final manuscript.

\section{Publisher's Note}

Springer Nature remains neutral with regard to jurisdictional claims in published maps and institutional affiliations.

Received: 3 January 2020 Accepted: 5 February 2021 Published online: 22 February 2021

\section{References}

1. Cao, J., Feng, G., Wang, Y.: Multistability and multiperiodicity of delayed Cohen-Grossberg neural networks with a general class of activation functions. Physica D 237(13), 1734-1749 (2008) 
2. Cheng, C., Shi, C.: Complete stability in multistable delayed neural networks. Neural Comput. 21(3), 719-740 (2009)

3. Zhang, L., Yi, Z., Zhang, S., Heng, P.: Activity invariant sets and exponentially stable attractors of linear threshold discrete-time recurrent neural networks. IEEE Trans. Autom. Control 54(6), 1341-1347 (2009)

4. Nie, X., Cao, J.: Multistability of competitive neural networks with time-varying and distributed delays. Nonlinear Anal., Real World Appl. 10, 928-942 (2009)

5. Huang, Z., Song, Q. Feng, C.: Multistability in networks with self-excitation and high-order synaptic connectivity. IEEE Trans. Circuits Syst. I, Regul. Pap. 57(8), 2144-2155 (2010)

6. Zeng, Z., Huang, T., Zheng, W.: Multistability of recurrent neural networks with time-varying delays and the piecewise linear activation function. IEEE Trans. Neural Netw. 21(8), 1371-1377 (2010)

7. Huang, G., Cao, J.: Delay-dependent multistability in recurrent neural networks. Neural Netw. 23(2), 201-209 (2010)

8. Wang, L., Lu, W., Chen, T.: Coexistence and local stability of multiple equilibria in neural networks with piecewise linear nondecreasing activation functions. Neural Netw. 23, 189-200 (2010)

9. Kaslik, E., Sivasundaram, S.: Impulsive hybrid discrete-time Hopfield neural networks with delays and multistability analysis. Neural Netw. 24(4), 370-377 (2011)

10. Nie, X., Cao, J.: Multistability of second-order competitive neural networks with nondecreasing saturated activation functions. IEEE Trans. Neural Netw. 22(11), 1694-1708 (2011)

11. Wang, L., Chen, T:: Complete stability of cellular neural networks with unbounded time-varying delays. Neural Netw. 36, 11-17 (2012)

12. Zeng, Z., Zheng, W.: Multistability of neural networks with time-varying delays and concave-convex characteristics. IEEE Trans. Neural Netw. Learn. Syst. 23(2), 293-305 (2012)

13. Marco, M., Forti, M., Grazzini, M., Pancioni, L.: Limit set dichotomy and multistability for a class of cooperative neural networks with delays. IEEE Trans. Neural Netw. Learn. Syst. 23(9), 1473-1485 (2012)

14. Wang, L., Chen, T.: Multistability of neural networks with Mexican-hat-type activation functions. IEEE Trans. Neural Netw. Learn. Syst. 23(11), 1816-1826 (2012)

15. Du, Y., Li, Y., Xu, R.: Multistability and multiperiodicity for a general class of delayed Cohen-Grossberg neural networks with discontinuous activation functions. Discrete Dyn. Nat. Soc. 917835, 1-11 (2013)

16. Wang, L., Chen, T: Multiple $\mu$-stability of neural networks with unbounded time-varying delays. Neural Netw. 53 , 109-118 (2014)

17. Cheng, C., Huang, Z:: Non-typical multistability in neural networks with distributed delays. Neurocomputing 121 207-217 (2013)

18. Zhou, B., Song, Q.: Boundedness and complete stability of complex-valued neural networks with time delay. IEEE Trans. Neural Netw. Learn. Syst. 24(8), 1227-1238 (2013)

19. Huang, Z., Raffoul, Y., Cheng, C.: Scale-limited activating sets and multiperiodicity for threshold networks on time scales. IEEE Trans. Cybern. 44(4), 488-499 (2014)

20. Nie, X., Zheng, W.: Multistability and instability of neural networks with discontinuous nonmonotonic piecewise linear activation functions. IEEE Trans. Neural Netw. Learn. Syst. 26(11), 2901-2913 (2015)

21. Nie, X., Zheng, W.: Multistability of neural networks with discontinuous non-monotonic piecewise linear activation functions and time-varying delays. Neural Netw. 65, 65-79 (2015)

22. Nie, X., Zheng, W.: Complete stability of neural networks with nonmonotonic piecewise linear activation functions. IEEE Trans. Circuits Syst. II, Express Briefs 62(10), 1002-1006 (2015)

23. Nie, X., Zheng, W., Cao, J.: Coexistence and local $\mu$-stability of multiple equilibrium points for memristive neural networks with nonmonotonic piecewise linear activation functions and unbounded time-varying delays. Neural Netw. 84, 172-180 (2016)

24. Yang, W., Wang, Y., Zeng, Z., Zheng, D.: Multistability of discrete-time delayed Cohen-Grossberg neural networks with second-order synaptic connectivity. Neurocomputing 164, 252-261 (2015)

25. Liang, J., Gong, W., Huang, T.: Multistability of complex-valued neural networks with discontinuous activation functions. Neural Netw. 84, 125-142 (2016)

26. Liu, P., Zeng, Z., Wang, J.: Multistability analysis of a general class of recurrent neural networks with non-monotonic activation functions and time-varying delays. Neural Netw. 79, 117-127 (2016)

27. Liu, P., Zeng, Z., Wang, J.: Multistability of recurrent neural networks with non-monotonic activation functions and mixed time delays. IEEE Trans. Syst. Man Cybern. 46(4), 512-523 (2016)

28. Chen, X., Zhao, Z., Song, Q., Hu, J.: Multistability of complex-valued neural networks with time-varying delays. Appl. Math. Comput. 294, 18-35 (2017)

29. Tan, M., Xu, D.: Multiple $\mu$-stability analysis for memristor-based complex-valued neural networks with nonmonotonic piecewise nonlinear activation functions and unbounded time-varying delays. Neurocomputing $275,2681-2701(2018)$

30. Nie, X., Liang, W., Cao, J.: Multistability analysis of competitive neural networks with Gaussian-wavelet-type activation functions and unbounded time-varying delays. Appl. Math. Comput. 356, 449-468 (2019)

31. Nie, X., Liang, W., Cao, J: Multistability and instability of competitive neural networks with non-monotonic piecewise linear activation functions. Nonlinear Anal., Real World Appl. 45, 799-821 (2019)

32. Chen, Y., Jia, S.: Multiple stability and instability of Cohen-Grossberg neural network with unbounded time-varying delays. J. Inequal. Appl. 178, 1-14 (2019)

33. Chen, J., Chen, B., Zeng, Z., Jiang, P.: Event-based synchronization for multiple neural networks with time delay and switching disconnected topology. IEEE Trans. Cybern. 99, 1-11 (2020)

34. Huang, Y., Yuan, X., Yang, X., Long, H.: Multiple Lagrange stability and Lyapunov asymptotical stability of delayed fractional-order Cohen-Grossberg neural networks. Chin. Phys. B 29, 229-238 (2020)

35. Wan, P., Sun, D., Zhao, M., Wan, L., Jin, S.: Multistability and attraction basins of discrete-time neural networks with non-monotonic piecewise linear activation functions. Neural Netw. 122, 231-238 (2020)

36. Agranovich, G., Litsyn, E., Slavova, A.: Impulsive control of a hysteresis cellular neural network model. Nonlinear Anal. Hybrid Syst. 3, 65-73 (2009)

37. Lu, J., Kurths, J., Cao, J., et al.: Synchronization control for nonlinear stochastic dynamical networks: pinning impulsive strategy. IEEE Trans. Neural Netw. 23, 285-292 (2012) 
38. Yang, X., Cao, J., Qiu, J.: p-moment exponential stochastic synchronization of coupled memristor-based neural networks with mixed delays via delayed impulsive control. Neural Netw. 65, 80-91 (2015)

39. Liu, X., Wang, Q.: Impulsive stabilization of high-order Hopfield-type neural networks with time-varying delays. IEEE Trans. Neural Netw. 19,71-79 (2008)

40. Li, X., Regan, D., Akca, H.: Global exponential stabilization of impulsive neural networks with unbounded continuously distributed delays. IMA J. Appl. Math. 80, 85-99 (2015)

41. Li, L., Jian, J.: Delay-dependent passivity analysis of impulsive neural networks with time-varying delays. Neurocomputing 168, 276-282 (2015)

42. Guan, Z., Liu, Z., Feng, G., et al.: Synchronization of complex dynamical networks with time-varying delays via impulsive distributed control. IEEE Trans. Circuits Syst. I, Regul. Pap. 57, 2182-2195 (2010)

43. Guan, Z., Hill, D., Shen, X.: Hybrid impulsive and switching systems and application to control and synchronization. IEEE Trans. Autom. Control 50, 1058-1062 (2005)

44. Chen, W., Lu, X., Zheng, W.: Impulsive stabilization and impulsive synchronization of discrete-time delayed neural networks. IEEE Trans. Neural Netw. 26, 734-748 (2015)

45. Feng, Y., Li, C., Huang, T.: Sandwich control systems with impulse time windows. Int. J. Mach. Learn. Cybern. 8 , 2009-2015 (2017)

46. Li, X., Zhang, X., Song, S.: Effect of delayed impulses on input-to-state stability of nonlinear systems. Automatica 76 , 378-382 (2017)

47. Stamova, I., Stamov, T., Li, X.: Global exponential stability of a class of impulsive cellular neural networks with supremums. Int. J. Adapt. Control Signal Process. 28, 1227-1239 (2014)

48. Yao, F., Deng, F.: Stability of impulsive stochastic functional differential systems in terms of two measures via comparison approach. Sci. China Inf. Sci. 55, 1313-1322 (2012)

49. Li, C., Wu, S., Feng, G., et al.: Stabilizing effects of impulses in discrete-time delayed neural networks. IEEE Trans. Neural Netw. 22, 323-329 (2011)

50. Li, X., Song, S., Wu, J.: Impulsive control of unstable neural networks with unbounded time-varying delays. Sci. China Inf. Sci. 61, 012203 (2018)

51. Khan, H., Khan, A., Jarad, F., Shah, A.: Existence and data dependence theorems for solutions of an ABC-fractional order impulsive system. Chaos Solitons Fractals 131, 109477 (2020)

52. Khan, H., Khan, A., Abdeljawad, T., Alkhazzan, A.: Existence results in Banach space for a nonlinear impulsive system. Adv. Differ. Equ. 2019, 18 (2019)

\section{Submit your manuscript to a SpringerOpen ${ }^{\circ}$ journal and benefit from:}

- Convenient online submission

- Rigorous peer review

- Open access: articles freely available online

- High visibility within the field

- Retaining the copyright to your article

Submit your next manuscript at $\boldsymbol{~ s p r i n g e r o p e n . c o m ~}$ 